\title{
Superconducting Cavity Control and Model Identification based on Active Disturbance Rejection Control
}

\author{
Zheqiao Geng, Member, IEEE
}

\begin{abstract}
Superconducting cavities are widely used in linear accelerator facilities all over the world. It is challenging for the Low Level RF (LLRF) system to stabilize the RF field inside the cavities working in pulsed mode with heavy beam loading and strong Lorenz force detuning. Feedback loops are implemented in LLRF system which should have fast responses to the disturbances on cavities. To enhance the feedback performance, active compensation of Lorenz force detuning with Piezo tuners and the compensation of beam loading with feed forward control are strongly needed. They all require the knowledge of cavity parameters, such as the quality factor, time varying detuning and the amplitude and phase of the beam. A disturbance observer based on the Active Disturbance Rejection Control (ADRC) was designed for superconducting cavities counting the Lorenz force detuning and beam loading as disturbances to the cavities. The ADRC disturbance observer can be integrated into LLRF feedback loops to speed up the responses to disturbances and the outputs of the observer are interpreted furtherly to identify the cavity parameters. This paper presents the algorithm and architecture of the ADRC disturbance observer, the simulation of the LLRF feedback loop with disturbance rejection and the cavity parameter identification applied on the archived data of superconducting cavities in operation.
\end{abstract}

Index Terms-LLRF, Superconducting Cavity, Disturbance Observer, Active Disturbance Rejection Control, Feedback, System Identification

\section{INTRODUCTION}

$S^{\mathrm{u}}$ perconducting cavies are used in many accelerator facilities either already in operation or still under construction, such as the European X-ray Free Electron Laser (XFEL) at DESY, the Linac Coherent Light Source II (LCLS-II) under construction at SLAC and the European Spallation Source (ESS) under construction at ESS. In these facilities, the superconducting cavities are used to accelerate either electron or proton beams in pulsed or Continuous-Wave (CW) modes.

In order to produce high quality beams, especially in the FEL

Manuscript received November 21, 2016.

Z. Geng was with the Beam Controls Group, Deutsches ElektronenSynchrotron DESY, Notkestraße 85, 22607 Hamburg, Germany. He is now with the LLRF section, Paul Scherrer Institut, 5232 Villigen PSI, Switzerland (phone: +41 7886380 59; e-mail: zheqiao.geng@psi.ch). machines, the amplitude and phase stabilities of the RF fields in superconducting cavities are very important. LLRF systems are typically employed to detect and stabilize the RF fields in cavities [1-3].

In superconducting cavities, the major sources to disturb the RF fields include:

1) Cavity detuning caused by microphonics and Lorenz force. When the cavity is detuned, more RF power is needed to keep the cavity voltage and the reflection power is increased. A random detuning caused by microphonics (e.g. vibrations due to ground motions, helium flows and water pumps) introduces amplitude and phase fluctuations in cavities.

2) Beam loading. The beam loading effects in superconducting cavities are normally unneglectable which generate repetitive errors in RF fields. Fluctuations of beam current also generate RF amplitude and phase fluctuations.

In order to well maintain the RF stabilities, the LLRF system should provide some specific features. A low noise low drift RF detector should be designed to measure the RF fields seen by beam accurately and precisely. A feedback controller should be able to provide fast responses to the perturbations on RF system. Feed forward control can be introduced to remove the repetitive field errors especially for the RF stations operated in pulsed mode. Resonance frequency control to compensate the Lorenz force detuning and microphonics helps to reduce the required RF power and improve the RF stabilities. A general control scheme for superconducting cavities is summarized in Fig. 1.

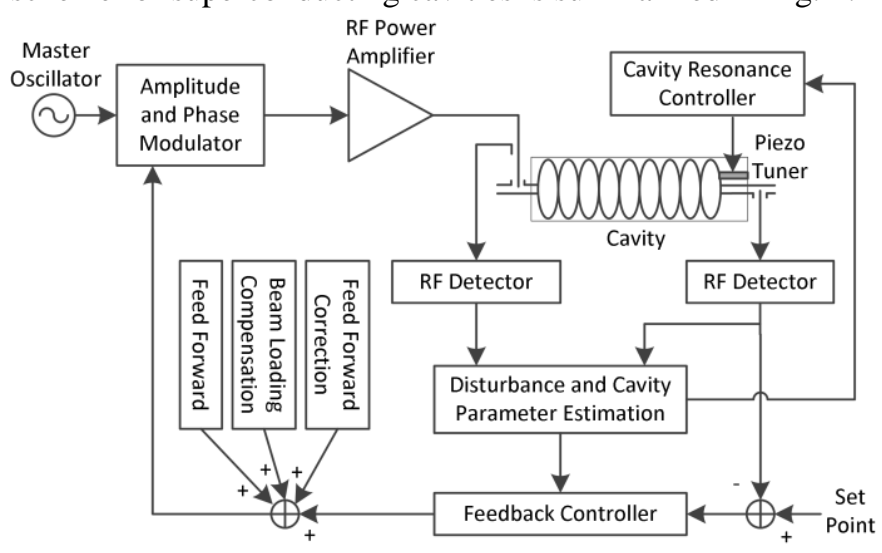

Fig. 1. A general control scheme of superconducting cavities.

In Fig. 1, an RF based feedback loop is employed to stabilize 
the RF fields in the cavity. The cavity probe signal is measured with an RF detector, which typically contains a down converter and an ADC. The feedback controller is used to generate RF drive signals according to the errors in RF fields compared with the set point. A general feed forward signal is used to define the working point of the RF station including the shapes of RF pulses. A beam loading compensation signal is applied to roughly compensate the beam loading when switching on the beam. Repetitive errors caused by Lorenz force detuning and beam loading are furtherly compensated with adaptive algorithms like Iterative Learning Control (ILC) [4]. In parallel to the RF based feedback loop, the cavity resonance control loop is used to actively compensate the Lorenz force detuning and microphonics [5-6]. The detuning of the cavity is estimated with the cavity RF signals and used as an input to the resonance control loop.

The RF feedback controller is the key part to suppress the random errors in RF fields. It should have fast responses to disturbances and should be able to work reliably. The Proportional-Integral (PI) controller is widely used in LLRF systems [7-8]. It is simple, reliable and easy to understand. But the performance is quite limited especially with fast disturbances. In several LLRF systems, the PI controllers were extended with different kinds of disturbance observers [9-10] to improve the disturbance rejection capability of the feedback controllers. Particularly, the ADRC based feedback controller has been successfully applied to the control of superconducting cavities [10] which showed attractive performance with simple implementations. ADRC is a control strategy inherits from the Proportional-Integral-Derivative (PID) control [11-12]. It actively estimates the disturbances to the system in real-time with a state observer and mitigates them with an extra input to the system in addition to the input generated by the PID controller. The uncertainties of the system model are also included as part of the estimated disturbances and mitigated by the disturbance rejection which makes ADRC a robust control method [13-17]. ADRC can be applied not only on linear systems (e.g. the superconducting cavities discussed in this paper) but also nonlinear systems [18-19].

In addition to feedbacks, the performance of RF controller can be improved by integrating the functions of cavity resonance control and beam loading compensation. Then the cavity parameters, such as the quality factor, the time varying detuning during RF pulse and the magnitude and phase of the beam loading need to be identified during run-time of the cavity. If the beam loading and cavity detuning are viewed as disturbances to the system, they can be identified from the output of the disturbance observer implemented for the feedback controller.

In this paper, a disturbance observer based on ADRC was derived for the superconducting cavity described with a multiple-input multiple-output (MIMO) model. The major goal for this paper is to interpret the output of the ADRC disturbance observer according to the cavity model to identity the cavity parameters like beam loading, quality factor and detuning. The results can be used as inputs for cavity resonance control and beam loading compensation. The feedback controller based on ADRC was also studied with simulations to complete the description of a systematic strategy for superconducting cavity control.

\section{CAVITY DISTURBANCE OBSERVER}

\section{A. Cavity Model}

A superconducting cavity is normally driven by an RF power amplifier such as a solid state amplifier or a klystron. The RF power is transmitted by a transmission line and coupled into the cavity with a coupler. The cavity fields will also be influenced by a beam current which appears as the second source of RF fields as shown in Fig. 2.

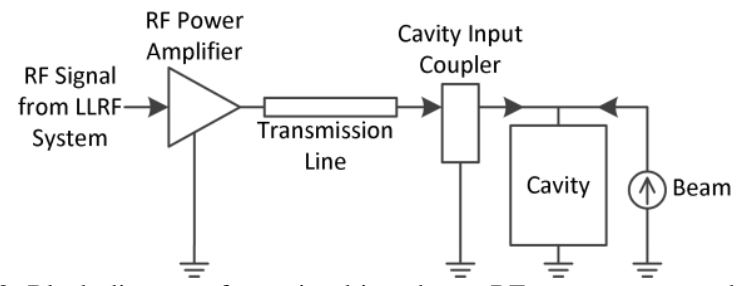

Fig. 2. Block diagram of a cavity driven by an RF power source and beam loading.

The behavior of the cavity represented by the envelope of the $\mathrm{RF}$ fields can be described by a first-order complex differential equation [20]:

$$
\dot{\mathbf{V}}_{c}+\left(\omega_{1 / 2}-j \Delta \omega\right) \mathbf{V}_{c}=2 \omega_{1 / 2}\left(\mathbf{V}_{r f}+\mathbf{V}_{b}\right),
$$

where $\mathbf{V}_{c}$ is the phasor representing the cavity voltage, $\omega_{1 / 2}$ is the half-bandwidth of the cavity and $\Delta \omega$ is the detuning, $\mathbf{V}_{r f}$ and $\mathbf{V}_{b}$ are effective driving voltage phasors for RF power and beam loading. The factor 2 at the right side comes from the fact that the coupling factor of superconducting cavities is much larger than 1 resulting in double voltage in the cavity compared to the driving voltage [21]. $\mathbf{V}_{r f}$ is the driving voltage caused by the RF power applying on the resistance of the cavity through the input coupler. $\mathbf{V}_{b}$ is the voltage generated by the DC component of the beam current applying on the cavity resistance. Equation (1) is the basis for the discussions in the following parts of the paper.

\section{B. ADRC Disturbance Observer}

In the existing design based on ADRC [10], the cavity model was separated as two single-input single-output (SISO) systems by decoupling the real and imaginary parts of the complex equation (1), and then ADRC observers were defined separately for the two decoupled SISO systems. In this paper, the MIMO cavity model will be directly used and a single ADRC observer will be designed. Such treatment of the ADRC observer will be helpful to identify the cavity parameters which are usually presented as complex numbers.

The MIMO cavity equation can also be derived from (1) by separating the real (with $r$ in subscript) and imaginary (with $i$ in subscript) parts but still keeping the matrix form of the equation:

$$
\left[\begin{array}{c}
\dot{V}_{c r} \\
\dot{V}_{c i}
\end{array}\right]=\left[\begin{array}{cc}
-\omega_{1 / 2} & -\Delta \omega \\
\Delta \omega & -\omega_{1 / 2}
\end{array}\right]\left[\begin{array}{c}
V_{c r} \\
V_{c i}
\end{array}\right]+2 \omega_{1 / 2}\left[\begin{array}{c}
V_{r f r} \\
V_{r f i}
\end{array}\right]+2 \omega_{1 / 2}\left[\begin{array}{c}
V_{b r} \\
V_{b i}
\end{array}\right] .
$$


Define the terms

$$
\begin{array}{ll}
\mathbf{y}=\left[\begin{array}{l}
V_{c r} \\
V_{c i}
\end{array}\right], \quad \mathbf{A}=\left[\begin{array}{cc}
-\omega_{1 / 2} & -\Delta \omega \\
\Delta \omega & -\omega_{1 / 2}
\end{array}\right], \\
b_{0}=2 \omega_{1 / 2}, \quad \mathbf{u}=\left[\begin{array}{c}
V_{r f r} \\
V_{r f i}
\end{array}\right], \quad \mathbf{d}=\left[\begin{array}{c}
V_{b r} \\
V_{b i}
\end{array}\right]
\end{array}
$$

where the beam loading is viewed as a disturbance $\mathbf{d}$, the cavity equation can be rewritten as

$$
\dot{\mathbf{y}}=\mathbf{A y}+b_{0} \mathbf{u}+b_{0} \mathbf{d} \text {. }
$$

In equation (2), the detuning mainly caused by the Lorenz force and the mechanical dynamics of the cavity and the driving term caused by beam are usually unknown. They are normally viewed as part of disturbances to the cavity. The cavity driving term caused by RF is usually derived from the measurement of the RF signal at the cavity input with calibrations in amplitude and phase. The errors in the calibration of RF drive can also be viewed as a disturbance, which can be covered by introducing an error term $\Delta b$ in (4) by replacing $b_{0}$ before $\mathbf{u}$ with $b_{0}+\Delta b$. The half-bandwidth of the cavity $\omega_{1 / 2}$ can be accurately derived from the measurement of cavity probe signal so it is reasonable to assume that the uncertainty in $b_{0}$ is neglectable. With these considerations, the cavity equation can be written as

$$
\begin{aligned}
\dot{\mathbf{y}} & \approx \mathbf{A} \mathbf{y}+\left(b_{0}+\Delta b\right) \mathbf{u}+b_{0} \mathbf{d}=\left(\mathbf{A y}+\Delta b \mathbf{u}+b_{0} \mathbf{d}\right)+b_{0} \mathbf{u}, \\
& =\mathbf{f}+b_{0} \mathbf{u}
\end{aligned}
$$

where $\mathbf{f}$ is the general disturbance term which will be estimated by the ADRC observer. In the equations above, $\mathbf{y}, \mathbf{u}, \mathbf{d}$ and $\mathbf{f}$ are all 2-by-1 vectors containing real and imaginary parts.

The estimations of the cavity voltage and general disturbance term can be defined as

$$
\hat{\mathbf{y}}=\hat{\mathbf{x}}_{1}, \quad \hat{\mathbf{f}}=\hat{\mathbf{x}}_{\mathbf{2}} .
$$

An observer can be defined to estimate the two items in equation (6) based on the measurements of the input $\mathbf{u}$ and output $\mathbf{y}$ of the cavity:

$$
\left[\begin{array}{c}
\dot{\mathbf{x}}_{1} \\
\dot{\mathbf{x}}_{2}
\end{array}\right]=\left[\begin{array}{cc}
\mathbf{0} & \mathbf{I} \\
\mathbf{0} & \mathbf{0}
\end{array}\right]\left[\begin{array}{c}
\hat{\mathbf{x}}_{1} \\
\hat{\mathbf{x}}_{2}
\end{array}\right]+b_{0}\left[\begin{array}{l}
\mathbf{I} \\
\mathbf{0}
\end{array}\right] \mathbf{u}+\mathbf{L}\left(\mathbf{y}-\hat{\mathbf{x}}_{1}\right),
$$

where $\mathbf{L}$ is a 4-by-2 parameter matrix which need to be determined to implement the observer [22]. Equation (7) can be rewritten as

$$
\left[\begin{array}{c}
\dot{\hat{V}}_{c r} \\
\dot{\hat{V}}_{c i} \\
\dot{\hat{f}}_{r} \\
\dot{\hat{f}}_{i}
\end{array}\right]=\left[\begin{array}{llll}
-l_{11} & -l_{12} & 1 & 0 \\
-l_{21} & -l_{22} & 0 & 1 \\
-l_{31} & -l_{32} & 0 & 0 \\
-l_{41} & -l_{42} & 0 & 0
\end{array}\right]\left[\begin{array}{l}
\hat{V}_{c r} \\
\hat{V}_{c i} \\
\hat{f}_{r} \\
\hat{f}_{i}
\end{array}\right]+\left[\begin{array}{llll}
l_{11} & l_{12} & b_{0} & 0 \\
l_{21} & l_{22} & 0 & b_{0} \\
l_{31} & l_{32} & 0 & 0 \\
l_{41} & l_{42} & 0 & 0
\end{array}\right]\left[\begin{array}{l}
V_{c r} \\
V_{c i} \\
V_{d r} \\
V_{d i}
\end{array}\right] .
$$

The dynamics of the observer should be faster than the closed loop behavior of the cavity, so that the estimation and rejection of disturbances can be performed before they generate large perturbations in cavity fields. With a proportional controller applied on the cavity, the closed loop pole of the system is at the location of

$$
s_{C L}=-K_{p} \cdot \omega_{1 / 2},
$$

where $K_{p}$ is the proportional gain which can be determined by the desired suppression ratio of the perturbations on cavity fields (e.g. if the perturbations caused by microphonics need to be suppressed by 100 times, a gain of 100 should be selected). For a simplified implementation, all the poles of the observer can be placed at the same location as

$$
s_{O B S}=n \cdot s_{C L} \text {, }
$$

where $n$ with a typical value between 3 to 10 [17]. With the desired pole location for the observer, the value of matrix $\mathbf{L}$ can be estimated.

If the detuning term in the cavity equation is viewed as part of the disturbances, equation (8) can be simplified by putting all the coupling items between real and imaginary parts to 0 . For most cases, the behaviors of the real and imaginary parts of the cavity have the same dynamics. With these considerations, the following assumptions are reasonable:

$$
\begin{aligned}
& l_{12}=l_{21}=l_{32}=l_{41}=0 \\
& l_{11}=l_{22}=M_{1} \\
& l_{31}=l_{42}=M_{2}
\end{aligned} .
$$

With the assumptions above, the characteristic equation of the observer in equation (8) can be presented as

$$
\lambda^{4}+\left(2 M_{1}\right) \lambda^{3}+\left(M_{1}^{2}+2 M_{2}\right) \lambda^{2}+\left(2 M_{1} M_{2}\right) \lambda+M_{2}^{2}=0 .
$$

If all the four poles of the observer are at the same location as in equation (10), the expected characteristic equation is

$$
\left(\lambda-s_{O B S}\right)^{4}=\lambda^{4}-4 s_{O B S} \lambda^{3}+6 s_{O B S}^{2} \lambda^{2}-4 s_{O B S}^{3} \lambda+s_{O B S}^{4}=0 .
$$

And then the parameter matrix $\mathbf{L}$ can be determined by comparing equation (12) and (13):

$$
M_{1}=-2 s_{O B S}, M_{2}=s_{O B S}^{2} .
$$

\section{Feedback with ADRC}

With the observer, the cavity voltage $\hat{\mathbf{y}}$ and disturbance $\hat{\mathbf{f}}$ can be estimated and a controller with proportional control and disturbance rejection [10][17] can be designed with the control law:

$$
\mathbf{u}=\frac{\mathbf{K}_{\mathbf{p}} \cdot(\mathbf{r}-\hat{\mathbf{y}})-\hat{\mathbf{f}}}{b_{0}},
$$

where $\mathbf{K}_{\mathbf{p}}$ is the 2-by-2 gain matrix and $\mathbf{r}$ is the set point of the cavity voltage in real and imaginary format. The block diagram of the controller is shown in the figure below.

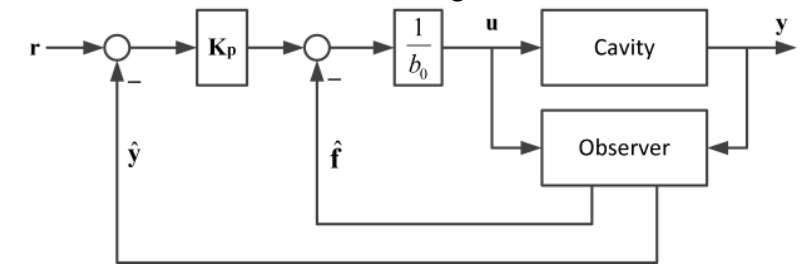

Fig. 3. Feedback loop with ADRC.

Simulations were used to compare the results of a simple proportional controller and the controller with disturbance rejection. The following parameters were used in the cavity model. 
TABLE I

PARAMETERS OF CAVITY SIMULATION

\begin{tabular}{ll}
\hline \multicolumn{1}{c}{ Parameter } & \multicolumn{1}{c}{ Value } \\
\hline Cavity r/Q & $1036 \Omega$ \\
Cavity loaded quality factor & $3 \mathrm{e} 6$ \\
RF frequency & $1.3 \mathrm{GHz}$ \\
Desired cavity voltage & $25 \mathrm{MV}$ \\
Beam current & $8 \mathrm{~mA}$ \\
Beam present time & $510 \mu$ s to $710 \mu \mathrm{s}$ \\
\hline \hline
\end{tabular}
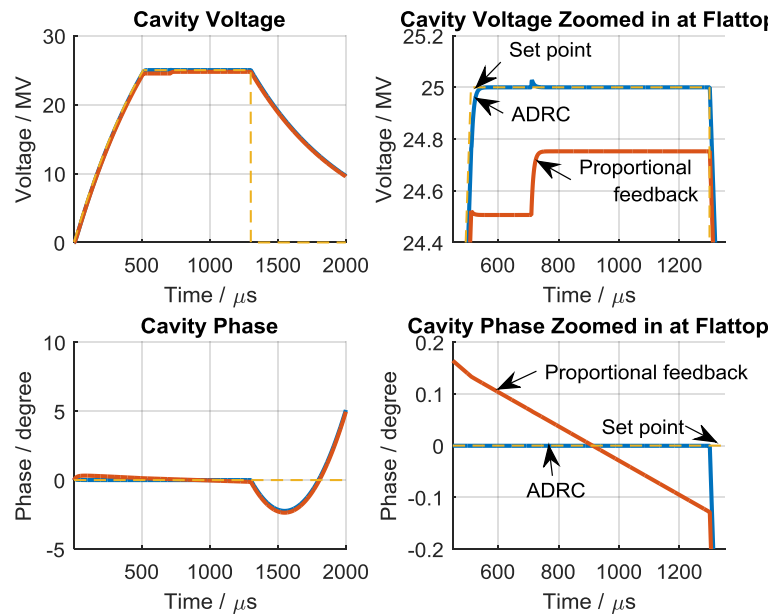

Fig. 4. Cavity voltage and phase waveforms in closed loop simulation of the two feedback strategies. The feedback loop based on ADRC showed better suppression of errors caused by Lorenz force detuning and beam loading.

In the simulation, Lorenz force detuning and beam loading were added to the cavity model to check the effects of the feedback loop with disturbance rejection. For both control loops, the same parameters were used including the same feedback gain as 100. For the feedback loop with disturbance rejection, the pole of the observer was 10 times larger in magnitude than the closed loop pole of the cavity. The amplitude and phase waveforms of the cavity in one RF pulse were plotted in Fig. 4 to compare the outputs of the two different controllers.

The output of the ADRC disturbance observer is shown in Fig. 5, from which the shapes of Lorenz force detuning and beam loading pulses are clearly visible. This led to the consideration to identify the cavity parameters, including the beam loading, with the estimated disturbance.
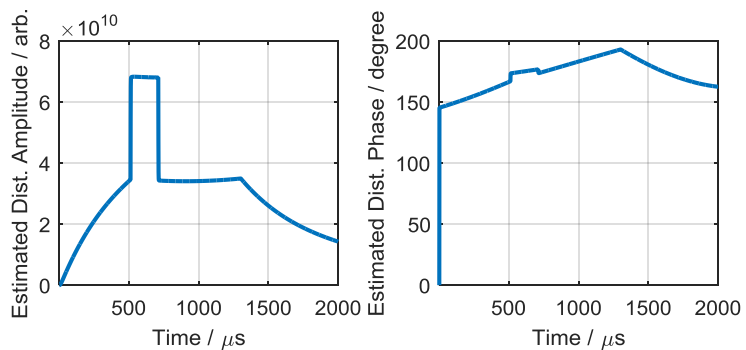

Fig. 5. Estimation of the disturbance term in amplitude and phase forms.

\section{CAVITY MODEL IDENTIFICATION}

\section{A. Overview}

For a practical RF station with superconducting cavities, the
RF signals picked up from the transmission line before the cavity input coupler and from the cavity probe are normally measured by RF detectors (see Fig. 1). The cavity model parameters can be identified with the available RF signal measurements and the results of the ADRC disturbance observer, which is depicted in Fig. 6 below.

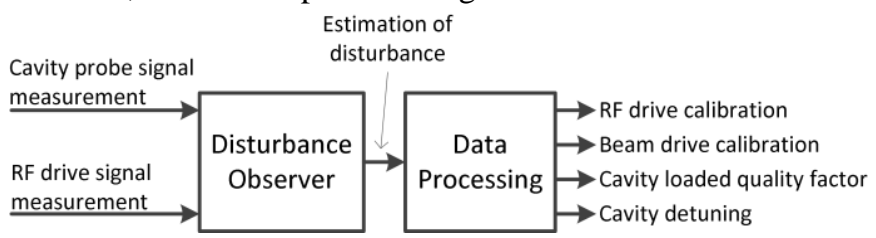

Fig. 6. Process of cavity model identification with disturbance observer.

The inputs of the process in Fig. 6 are in-phase (I) and quadrature $(\mathrm{Q})$ waveforms of the cavity probe signal and $\mathrm{RF}$ drive signal measured by the RF detectors with arbitrary attenuations and phase shifts in signal paths. The outputs of the process are the expected information about the cavity characteristics for the cavity model:

1) RF drive calibration: In order to apply the measurements of cavity probe and RF drive signals in the ADRC disturbance observer in equation (8), the arbitrary attenuations and phase shifts of the two channels should be calibrated. Normally the cavity probe signal is used as reference and the RF drive signal is calibrated with a complex factor.

2) Beam drive calibration: The magnitude and phase of the beam loading with respect to RF will be identified, which can be used to initialize the beam loading compensation, correct the drift in beam phase caused by the drifts in RF system and calibrate the cavity voltage, forward and reflected power if the beam current is accurately measured by beam diagnostics.

3) Cavity loaded quality factor: It is useful for quench detection in superconducting cavities.

4) Cavity detuning: It can be used as input to the cavity resonance controller.

The disturbance term introduced in equation (5) can be rewritten with the outputs of the ADRC disturbance observer as

$$
\hat{\mathbf{f}}=\mathbf{A} \hat{\mathbf{y}}+\Delta \mathbf{\Delta b} \cdot \mathbf{u}+b_{0} \mathbf{d} \text {. }
$$

The term $\Delta \mathbf{b}$ is a complex number representing the gain and phase uncertainties in the measurement path of cavity drive RF signal. Then equation (16) can be rewritten as

$$
\left[\begin{array}{l}
\hat{f}_{r} \\
\hat{f}_{i}
\end{array}\right]=\left[\begin{array}{cc}
-\omega_{1 / 2} & -\Delta \omega \\
\Delta \omega & -\omega_{1 / 2}
\end{array}\right]\left[\begin{array}{c}
\hat{V}_{c r} \\
\hat{V}_{c i}
\end{array}\right]+\left[\begin{array}{cc}
\Delta b_{r} & -\Delta b_{i} \\
\Delta b_{i} & \Delta b_{r}
\end{array}\right]\left[\begin{array}{l}
V_{r f r} \\
V_{r f i}
\end{array}\right]+b_{0}\left[\begin{array}{c}
V_{b r} \\
V_{b i}
\end{array}\right] .
$$

With the results derived from the ADRC disturbance observer and the measurement of the cavity drive RF signal, the $\mathrm{RF}$ drive scale factor $(\boldsymbol{\Delta b})$, the driving term caused by beam $\left(\mathbf{V}_{b}\right)$, the bandwidth $\left(\omega_{1 / 2}\right)$ and detuning $(\Delta \omega)$ of the cavity can be identified.

In this paper, the algorithm to identify the cavity parameters operated in pulsed mode will be presented. Dedicated algorithms need to be developed for cavities operated in $\mathrm{CW}$ mode which are out of the scope of this paper. 


\section{B. RF Drive Calibration}

The RF drive calibration can be performed when there is no beam in the cavity, so that the last term on the right side of equation (17) vanishes. To identify the RF drive scale factor, the measurement of the klystron output signal can be used as the RF driving term in the ADRC disturbance observer, leading to $\mathbf{u}=\mathbf{V}_{k l y}=\left[\begin{array}{lll}V_{k l y r} & V_{k l y i}\end{array}\right]^{T}$. Then equation (17) can be rewritten as

$$
\left[\begin{array}{l}
\hat{f}_{r} \\
\hat{f}_{i}
\end{array}\right]=\left[\begin{array}{cc}
-\omega_{1 / 2} & -\Delta \omega \\
\Delta \omega & -\omega_{1 / 2}
\end{array}\right]\left[\begin{array}{c}
\hat{V}_{c r} \\
\hat{V}_{c i}
\end{array}\right]+\left[\begin{array}{cc}
\Delta b_{r} & -\Delta b_{i} \\
\Delta b_{i} & \Delta b_{r}
\end{array}\right]\left[\begin{array}{c}
V_{k l y r} \\
V_{k l y i}
\end{array}\right] \text {. }
$$

Apply equation (18) to the waveforms of $\hat{\mathbf{f}}, \hat{\mathbf{V}}_{c}$ and $\mathbf{V}_{k l y}$, the following relation can be derived for each point in the waveforms by cancelling the detuning item:

$$
a(n) \Delta b_{r}+b(n) \Delta b_{i}=c(n),
$$

where $n$ is the index of the points in the waveforms and

$$
\begin{aligned}
& a(n)=V_{k l y r}(n) \cdot \hat{V}_{c r}(n)+V_{k l y i}(n) \cdot \hat{V}_{c i}(n) \\
& b(n)=V_{k l y r}(n) \cdot \hat{V}_{c i}(n)-V_{k l y i}(n) \cdot \hat{V}_{c r}(n) \\
& c(n)=\hat{f}_{r}(n) \cdot \hat{V}_{c r}(n)+\hat{f}_{i}(n) \cdot \hat{V}_{c i}(n)+\omega_{1 / 2}\left(\hat{V}_{c r}^{2}(n)+\hat{V}_{c i}^{2}(n)\right)
\end{aligned}
$$

The cavity bandwidth $\omega_{1 / 2}$ has been assumed to be a constant during the RF pulse, which is normally true if the cavity does not quench, and it can be calculated from the decay of the RF pulse [21]. From equation (19) and (20), the term $\Delta \mathbf{b}$ can be calculated with linear fitting, resulting in the RF drive scale factor shown below to convert $\mathbf{V}_{k l y}$ to $\mathbf{V}_{r f}$ :

$$
\mathbf{V}_{r f}=\mathbf{K}_{r f} \cdot \mathbf{V}_{k l y} \text {, with } \mathbf{K}_{r f}=1+\frac{\Delta \mathbf{b}}{b_{0}} .
$$

The calibration factor $\mathbf{K}_{r f}$ is a complex number which scales and rotates the klystron output signal. The calibration procedure described above can be executed for several iterations to achieve better results, with each time applying the calibrated RF drive signal to the ADRC disturbance observer.

\section{Identification of Cavity Parameters}

After applying the calibrated RF drive signal to the ADRC disturbance observer, the disturbance term will be mainly caused by the unknown beam loading and detuning, then equation (17) can be simplified as

$$
\left[\begin{array}{l}
\hat{f}_{r} \\
\hat{f}_{i}
\end{array}\right]=\left[\begin{array}{cc}
-\omega_{1 / 2} & -\Delta \omega \\
\Delta \omega & -\omega_{1 / 2}
\end{array}\right]\left[\begin{array}{c}
\hat{V}_{c r} \\
\hat{V}_{c i}
\end{array}\right]+b_{0}\left[\begin{array}{c}
V_{b r} \\
V_{b i}
\end{array}\right] .
$$

In equation (22), there are three items contributing to the estimated disturbance during the RF pulse: cavity bandwidth, detuning and beam loading. As a good approximation, the cavity bandwidth can be viewed as a constant and the value calculated from the decay of RF pulse can be used. The Lorenz force detuning is time variant during the RF pulse and reaches to its peak value at the end of the RF flattop. The detuning caused by microphonics normally changes slowly and can be viewed as a constant during the RF pulse with a pulse length smaller than several microseconds. With these considerations, the following procedure can be used to identify the cavity parameters:

1) Calculate the detuning of cavity during the RF pulse with beam switched off. The detuning waveform calculated from multiple RF pulses can be averaged to filter out the microphonics. The averaged detuning waveform comes from the combined effects of Lorenz force and piezo tuner feed forward control, and both of them are normally constant for a certain shape of RF amplitude waveform.

2) Calculate the cavity drive term caused by beam with the averaged detuning waveform from step 1 if the RF pulse shape with beam is similar to the one without beam. This is usually true when applying an effective feedback controller (e.g. controller with disturbance rejection) to the cavity control. The extra detuning caused by microphonics can be taken into account by comparing the detuning measured with the phase slope at the decay of RF pulse [21].

With the process above, the cavity drive signal caused by beam can be calculated from equation (22) as

$$
\mathbf{V}_{b}=\frac{\hat{\mathbf{f}}+\left(\omega_{1 / 2}-j \Delta \omega\right) \cdot \hat{\mathbf{V}}_{\mathbf{c}}}{b_{0}}
$$

\section{Applications of Cavity Model}

The identified cavity parameters, including the beam, can be used as inputs to many applications to set up and optimize the LLRF system. They are summarized in the table below:

TABLE II

APPLICATIONS OF IDENTIFIED CAVITY PARAMETERS

\begin{tabular}{ll}
\hline \hline Parameter & \multicolumn{1}{c}{ Application } \\
\hline$\omega_{1 / 2}$ & Quench detection. \\
$\Delta \omega$ & Cavity resonance control. \\
$\mathbf{V}_{b}$ & Cavity voltage calibration; \\
& Cavity forward and reflected power calibration; \\
& Beam phase calibration; \\
& Cavity drive phase correction (phasing); \\
& Vector sum calibration; \\
& Beam loading compensation. \\
\hline \hline
\end{tabular}

The cavity loaded quality factor $Q_{L}$ can be calculated from the cavity resonance frequency $\omega_{0}$ and cavity bandwidth with the equation $Q_{L}=\omega_{0} /\left(2 \omega_{1 / 2}\right)$. A significant drop in $Q_{L}$ normally indicates that the superconducting cavity quenches.

The detuning waveform during the RF pulse can be used to define the feed forward control signal for the piezo tuner.

$\mathbf{V}_{b}$ is the voltage generated by the DC component of beam current on the cavity resistance, which can be written as

$$
\mathbf{V}_{b}=R_{L} \mathbf{I}_{b 0}, \quad \text { with } R_{L}=\frac{1}{2}(r / Q) Q_{L},
$$

where $R_{L}$ is the loaded resistance of the cavity, $r / Q$ is the normalized shunt impedance of the cavity which is typically a design value of the cavity, $\mathbf{I}_{b 0}$ is the average current of the bunch train (e.g. a $1 \mathrm{MHz}$ bunch train with $1 \mathrm{nC}$ per bunch results in an average current of $1 \mathrm{~mA}$ ). If the beam current can be accurately measured by the beam diagnostics, the beam induced drive voltage can be calculated in physical unit (e.g. MV). Then by comparing the relative magnitude between the cavity voltage and the beam induced drive voltage, the cavity voltage in physical unit can be calibrated.

In Fig 2, if viewed after the cavity input coupler, the cavity system can be viewed as a transmission line terminated with the cavity resistance. Then the following relation applies to the cavity voltage $\mathbf{V}_{c}$, forward signal $\mathbf{V}_{r f}$ and reflected signal $\mathbf{V}_{\text {reff }}$ : 


$$
\mathbf{V}_{c}=\mathbf{V}_{r f}+\mathbf{V}_{\text {refl }} \text {. }
$$

Because the cavity voltage can be measured and the forward signal can be calculated with equation (21), the cavity reflected signal can be derived from equation (25). With the same calibration as for the cavity voltage, the forward and reflected signals can be obtained with physical unit, and then the instant power of the forward and reflected signals during the RF pulse can be calculated with the equations below:

$$
P_{r f}=\frac{\left|\mathbf{V}_{r f}\right|^{2}}{2 R_{L}}, \quad P_{r e f l}=\frac{\left|\mathbf{V}_{r e f f}\right|^{2}}{2 R_{L}} .
$$

The term $\mathbf{V}_{b}$ is a vector which also contains phase information. By comparing the phase of $\mathbf{V}_{b}$ and $\mathbf{V}_{c}$, the beam phase can be calibrated with the following relation:

$$
\varphi_{b}=\pi-\left(\angle \mathbf{V}_{b}-\angle \mathbf{V}_{c}\right) \text {. }
$$

Here we have defined the beam phase is 0 when $\mathbf{V}_{b}$ and $\mathbf{V}_{c}$ have opposite phases (on-crest acceleration). The beam phase is defined negative if the cavity instant voltage is rising up when the beam is injected into the cavity [21].

For an RF station with multiple cavities driven by a single RF power source, the relative phases between different cavities should be adjusted so that the beam sees unique phase from all cavities. The adjustment can be performed by comparing the identified beam phases in the cavities.

In order to measure the integrated RF fields seen by beam from the multiple cavities driven by the same RF power source, the vector sum of the voltages in all cavities should be calibrated to remove the gain and phase uncertainties in the arbitrary pickup cables [23]. If the beam is lossless through the cavities, the beam induced drive signal $\mathbf{V}_{b n}(\mathrm{n}=1,2, \ldots \mathrm{N}$ for $\mathrm{N}$ cavities $)$ should be identical taking into account the variations of $r / Q$ of each cavity. Then if we use the probe signal of the first cavity as reference, the measurements of the probe signals of other cavities should be scaled and rotated with the factors below:

$$
g_{n}=\frac{R_{L n}\left|\mathbf{V}_{b 1}\right|}{R_{L 1}\left|\mathbf{V}_{b n}\right|}, \quad \phi_{n}=\angle \mathbf{V}_{b 1}-\angle \mathbf{V}_{b n},
$$

where $R_{L n}$ is the loaded resistance of the $n$th cavity.

The beam loading compensation feed forward signal for the RF station with multiple cavities can be initially determined as

$$
S_{B L C}=-\frac{\sum_{n=1}^{N} \mathbf{V}_{b n} \cdot g_{n} e^{j \phi_{n}}}{N} .
$$

The beam loading compensation signal showed above refers to the cavity drive term $\mathbf{V}_{\text {rf }}$ and it should be traced back to the DAC outputs from the LLRF controller by taking out the extra gain and phase calibration between the DAC outputs and $\mathbf{V}_{r f}$. Normally the beam loading compensation signal should be applied to the LLRF controller at the same RF pulse when the beam is switched on to avoid big transient in the cavity fields. And because the beam information is still unknown at this moment, the beam loading compensation signal can be assigned to be a simple square pulse estimated from the desired beam current, beam phase and beam pulse length. After the first RF pulse with beam, the beam loading signal can be refined with the identified beam drive term $\mathbf{V}_{b}$ as in equation (29). Of course, ILC can be adopted to cover the imperfections in the beam loading compensation signal.

\section{Algorithm Test}

\section{A. Identification of Cavity Model}

The algorithms developed in the last section were applied to the data of measurements of cavities at FLASH archived years ago. Fig. 7 shows the raw measurements of the probe and klystron output signals of cavity No. 5 of the first module (ACC1) which are used as inputs to the algorithm tests.
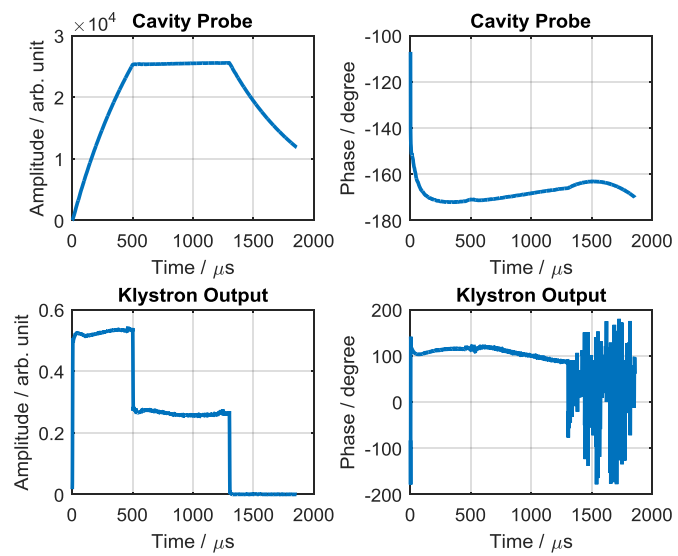

Fig. 7. Measurements of cavity probe and klystron output signal without beam. The klystron output signal is not calibrated. Results from decay: half bandwidth $=219.7 \mathrm{~Hz}$, detuning $=-20.2 \mathrm{~Hz}$.

After the RF drive calibration, the cavity drive signal induced by the klystron output power can be plotted as in Fig. 8 .
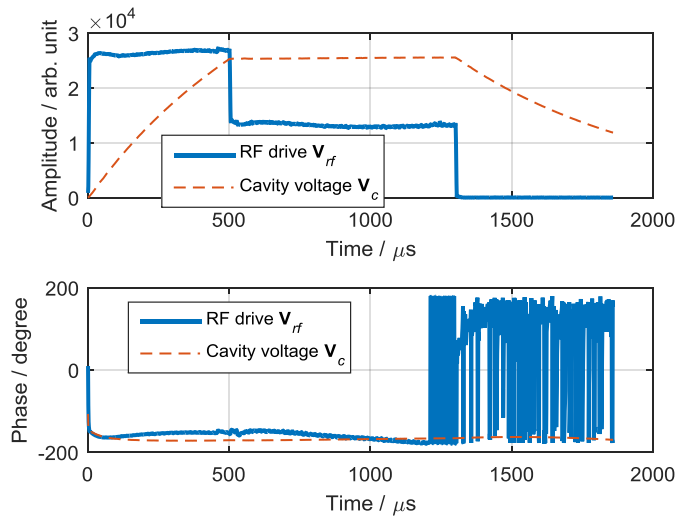

Fig. 8. RF drive signal with calibration. The cavity probe signal was used as reference for the calibration.

And because there was no beam presented in the cavity as the waveforms shown in Fig. 7, the detuning of the cavity can be directly identified by assuming the quality factor of the cavity as constant, which is shown in Fig. 12 as the reference detuning waveform. Later a beam pulse as in Fig. 9 was injected into the cavity. By applying the identification algorithm described in the last section, the beam induced cavity drive signal $\mathbf{V}_{b}$ was identified as in Fig. 10, from which the cavity voltage and beam phase could be calibrated. 


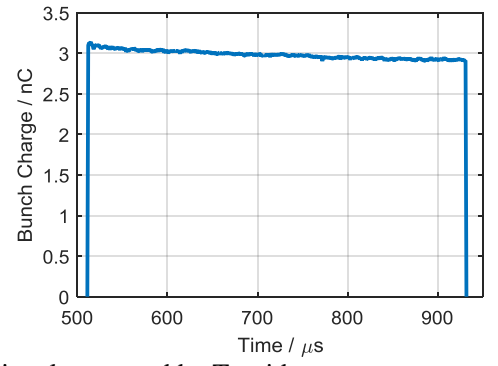

Fig. 9. Beam signal measured by Toroid.
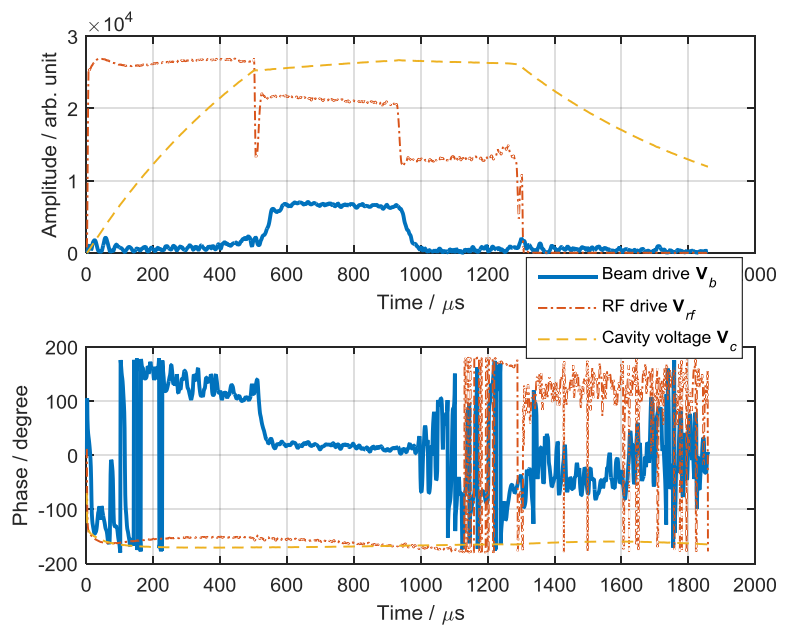

Fig. 10. Identified beam induced cavity drive signal. The RF power induced cavity drive signal and the cavity probe signal are also plotted as reference.

The amplitude and phase of $\mathbf{V}_{b}$ were identified for 100 pulses and the results are shown in Fig. 11.
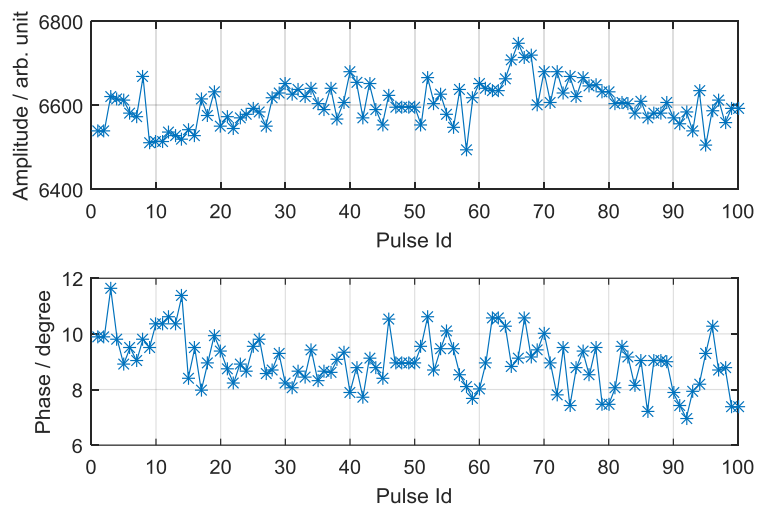

Fig. 11. 100 pulses identification of beam amplitude and phase averaged within the beam pulse. The amplitude jitter was $0.75 \%$ RMS and phase jitter 0.94 degree RMS. Lots of pulses should be averaged to get a good resolution for the beam phase measurement.

The cavity detuning waveform was then identified taking into account the beam.

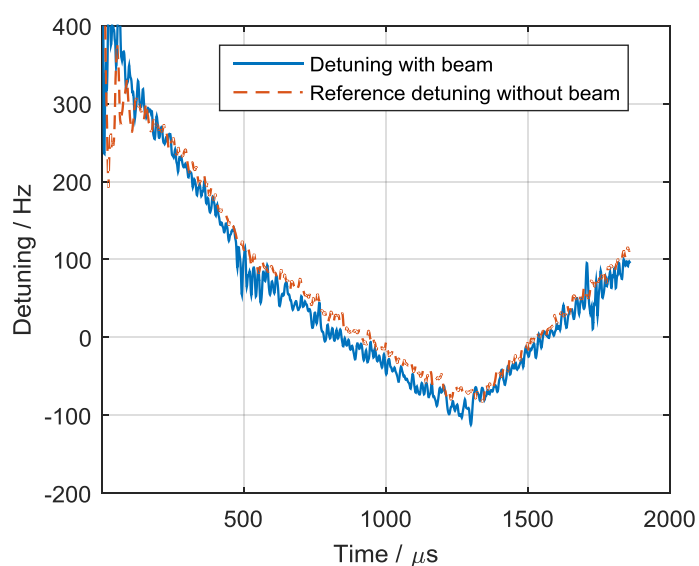

Fig. 12. Cavity detuning waveform with beam.

\section{B. RF Calibration}

With the beam information, the cavity voltage and beam phase can be calibrated, see Fig. 13.

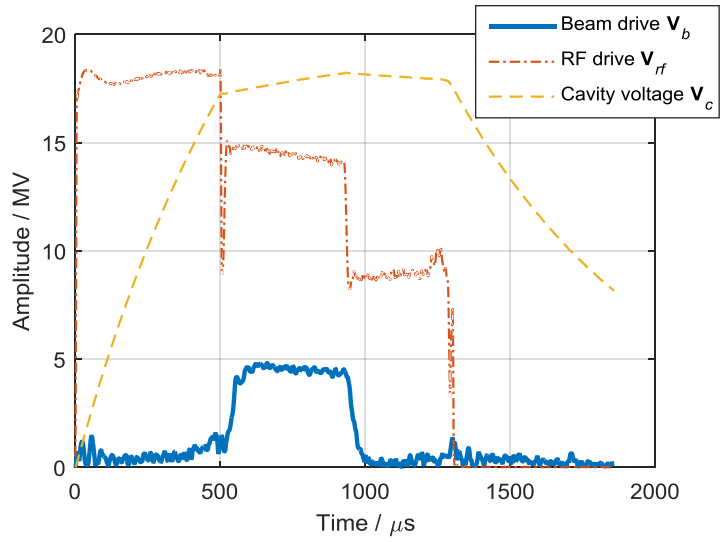

Fig. 13. Cavity voltage calibration with the beam current measurement and cavity design parameters $(r / Q)$. The beam phase is -11.35 degree.

Then the cavity driving power and reflected power can also be calibrated as in Fig. 14.

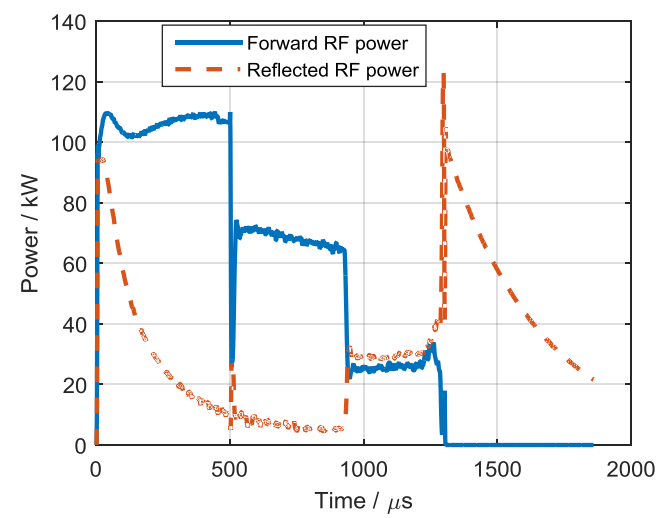

Fig. 14. Instant power of cavity forward and reflected signals.

\section{Error Analysis}

The detuning of the cavity without beam was used to decouple the contributions to disturbances from detuning and beam loading after the beam was switched on. The uncertainties in cavity detuning measurement will generate fluctuations in beam identification. The microphonics of the eight cavities of 
ACC1 were calculated for 100 pulses and then correlated with the jitters in the amplitude and phase of the identified beam induced drive signals, see Fig. 15.
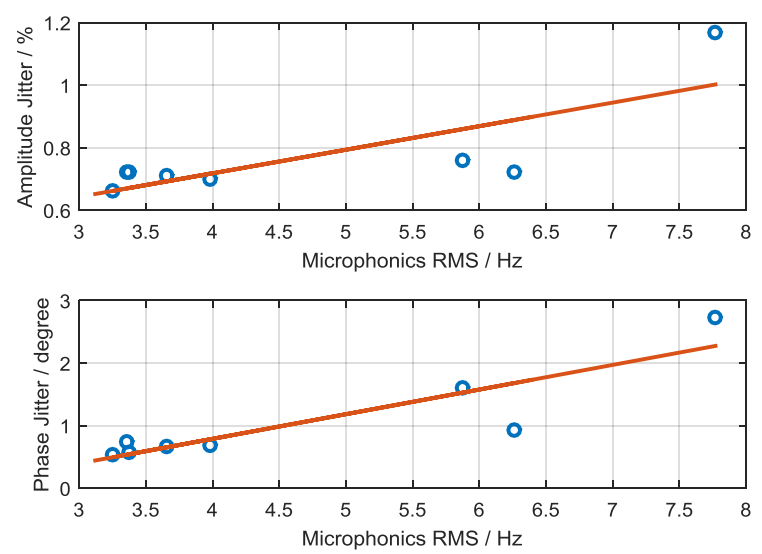

Fig. 15. Microphonics of eight cavities and the jitters of beam identification for 100 pulses. The sensitivity for amplitude jitter is $0.075 \% / \mathrm{Hz}$ and phase jitter 0.39 degree / Hz.

In order to get a precise measurement of beam phase and beam induced driving voltage, multiple pulses should be taken for averaging to reduce the noise.

\section{CONCLUSION}

The ADRC disturbance observer for superconducting cavities has a simple architecture with good understandability. The feedback controller based on ADRC shows very promising performance which can be an alternative to the existing controller with simple PI control. The output of the ADRC disturbance observer also provides a promising possibility to identify the cavity parameters during run-time which enables lots of applications to be implemented to calibrate, setup and optimize the LLRF system. A systematic control strategy for superconducting cavities based on the ADRC disturbance observer is foreseen by combining all the aspects like feedback controller, system identification and applications.

Generally, the same method can also be applied to other systems equipped with ADRC disturbance observers. By interpreting the output of the observer, the system parameters can be identified during run-time which can be used as inputs to on-line optimizations of the system parameters.

\section{REFERENCES}

[1] J. Branlard, G. Ayvazyan, V. Ayvazyan et al, "The European XFEL LLRF System," in Proc. IPAC2012 Conf., New Orleans, Louisiana, USA, 2012, pp. 55-57.

[2] L. Doolittle, G. Huang, A. Ratti et al, "The LCLS-II LLRF System," in Proc. IPAC2015 Conf., Richmond, VA, USA, 2015, pp. 1195-1197.

[3] Anders Johansson, Anders Svensson, Rihua Zeng et al, "LLRF System for the ESS Proton Accelerator," in Proc. IPAC2014 Conf., Dresden, Germany, 2014, pp. 2465-2467.

[4] Richard W. Longman, "Iterative Learning Control and Repetitive Control for Engineering Practice," Int. J. Control, vol. 73, pp. 930-954, Oct. 2000.

[5] K. Przygoda, T. Pozniak and M. Grecki, "Piezo Control for Lorenz Force Detuned SC Cavities of DESY FLASH," in Proc. IPAC10 Conf., Kyoto, Japan, 2010, pp. 1452-1454.
[6] Z.A. Conway and M.U. Liepe, "Fast Piezoelectric Actuator Control of Microphonics in the CW Cornell ERL Injector Cryomodule," in Proc. PAC09 Conf., Vancouver, BC, Canada, 2009, pp. 918-920.

[7] P. Fafara, W. Jalmuzna, W. Koprek et al, "FPGA-based Implementation of a Cavity Field Controller for FLASH and X-FEL," Meas. Sci. Technol., vol. 18, pp. 2365-2371, Jul. 2007.

[8] S. Michizono, H. Katagiri, T. Matsumoto et al, "Vector-sum Control of Superconducting RF Cavities at STF," in Proc. PAC09 Conf., Vancouver, BC, Canada, 2009, pp. 2204-2206.

[9] F. Qiu, D. Arakawa, Y. Honda et al, "A Disturbance-observer-based Controller for LLRF Systems," in Proc. IPAC2015 Conf., Richmond, VA, USA, 2015, pp. 2895-2898.

[10] J. Vincent, D. Morris, N. Usher et al, "On Active Disturbance Rejection Based Control Design for Superconducting RF Cavities," Nuclear Instruments and Methods in Physics Research A, vol. 643, pp. 11-16, Apr. 2011.

[11] J. Han, "Auto-disturbance rejection control and its applications," Control and Decision, vol. 13, pp. 19-23, Jan. 1998.

[12] J. Han, "From PID to Active Disturbance Rejection Control," IEEE Transactions on Industrial Electronics, vol. 56, pp. 900-906, March 2009.

[13] Z. Gao, Y. Huang and J. Han, "An Alternative Paradigm for Control System Design," Proc. of the 40th IEEE Conf. on Decision and Control, Florida, USA, 2001, pp. 4578-4585.

[14] Z. Gao, "Active Disturbance Rejection Control: A Paradigm Shift in Feedback Control System Design," Proc. of the 2006 American Control Conf., Minneapolis, USA, 2006, pp. 2399-2405.

[15] Q. Zhang and Z. Gao, "On Practical Applications of Active Disturbance Rejection Control," Proc. of the 29th Chinese Control Conf., Beijing, China, 2010, pp. 6095-6100.

[16] Q. Zhang, "On Active Disturbance Rejection Control: Stability Analysis and Applications in Disturbance Decoupling Control (Thesis or Dissertation style)," Ph.D. dissertation, Cleveland State University, Cleveland, USA, 2009.

[17] G. Herbst, "A Simulative Study on Active Disturbance Rejection Control (ADRC) as a Control Tool for Practitioners," Electronics, vol. 2, pp. 246-279, Aug. 2013.

[18] Z. Zhao and B. Guo, "On Active Disturbance Rejection Control for Nonlinear Systems Using Time-varying Gain," European Journal of Control, vol. 23, pp. 62-70, Feb. 2015.

[19] Q. Zheng, L. Gao and Z. Gao, "On Stability Analysis of Active Disturbance Rejection Control for Nonlinear Time-varying Plants with Unknown Dynamics," Proc. of the 46th IEEE Conf. on Decision and Control, New Orleans, Louisiana, USA, 2007, pp. 3501-3506.

[20] A. Brandt, "Development of a Finite State Machine for the Automated Operation of the LLRF Control at FLASH (Thesis or Dissertation style)," Ph.D. dissertation, Hamburg University, Hamburg, Germany, 2007.

[21] T. Schilcher, "Vector-sum Control of Pulsed Accelerating Fields in Lorenz Force detuned Superconducting Cavities (Thesis or Dissertation style)," Ph.D. dissertation, Hamburg University, Hamburg, Germany, 1998.

[22] R.C. Dorf and R.H. Bishop, Modern Control Systems. London, UK: Pearson Education, Inc., 2016.

[23] S.N. Simrock and T. Schilcher, "Transient Beam Loading based Calibration of the Vector-sum for the TESLA Test Facility," in Proc. EPAC96 Conf., Sitges, Spain, 1996, pp. 349-351. 